\title{
Apps galore
}

\section{A growing family of iPad and iPhone apps launched by publishers, institutes and societies active in photonics now makes it easy to keep up to date with the latest happenings in optics.}

Those of you who appreciate having electronic access to Nature Photonics when on the move will be pleased to hear that a new app for the iPad from the Nature Publishing Group now makes this easier than ever. The NatureJournals App makes it possible to download an entire issue of Nature Photonics (or any other Nature journal available in the app for which you have a subscription) that can then be conveniently browsed at your leisure - for example, when you are offline while travelling on a plane or train. An article can be bookmarked to facilitate retrieval at a later date, and news of its publication can be shared using social media tools like Facebook, Twitter and e-mail. Citation metrics and a summary of the article's figures can also be overlaid. Readers can choose between reading an article in the native app, which has the benefit of customizable font size and the above-mentioned functions, opening it as a PDF document or viewing it in the Safari web browser.

The app is available for free on Apple's iTunes store. Readers with an existing personal subscription to Nature Photonics simply need to $\log$ in to their nature.com account to gain access to all the journal's content, whereas non-subscribers are offered a free preview version that provides titles and abstracts of all the articles and free content from Nature news. A renewable one-month (30-day) subscription is available for US $\$ 4.99$; alternatively, an annual subscription can be purchased. Currently, access via site licences is not supported. Those with personal subscriptions to other Nature journals can access content from many other titles that also publish findings in photonics, including Nature, Nature Physics, Nature Materials, Nature Nanotechnology and Nature Communications. To help you stay up to date, the 'Latest Research' function in the app displays the details of papers from journals you have selected as they are published online. The app is compatible with any iPad running iOS 5 or later; a version for the iPhone is currently under development and should become available in early 2014 .

The NatureJournals App joins a growing family of free apps from organizations that are active in photonics. For example, the UK's Institute of Physics (IOP) Publishing has launched an app called IOPscience Express for the iPhone/iPod/iPad. This app provides

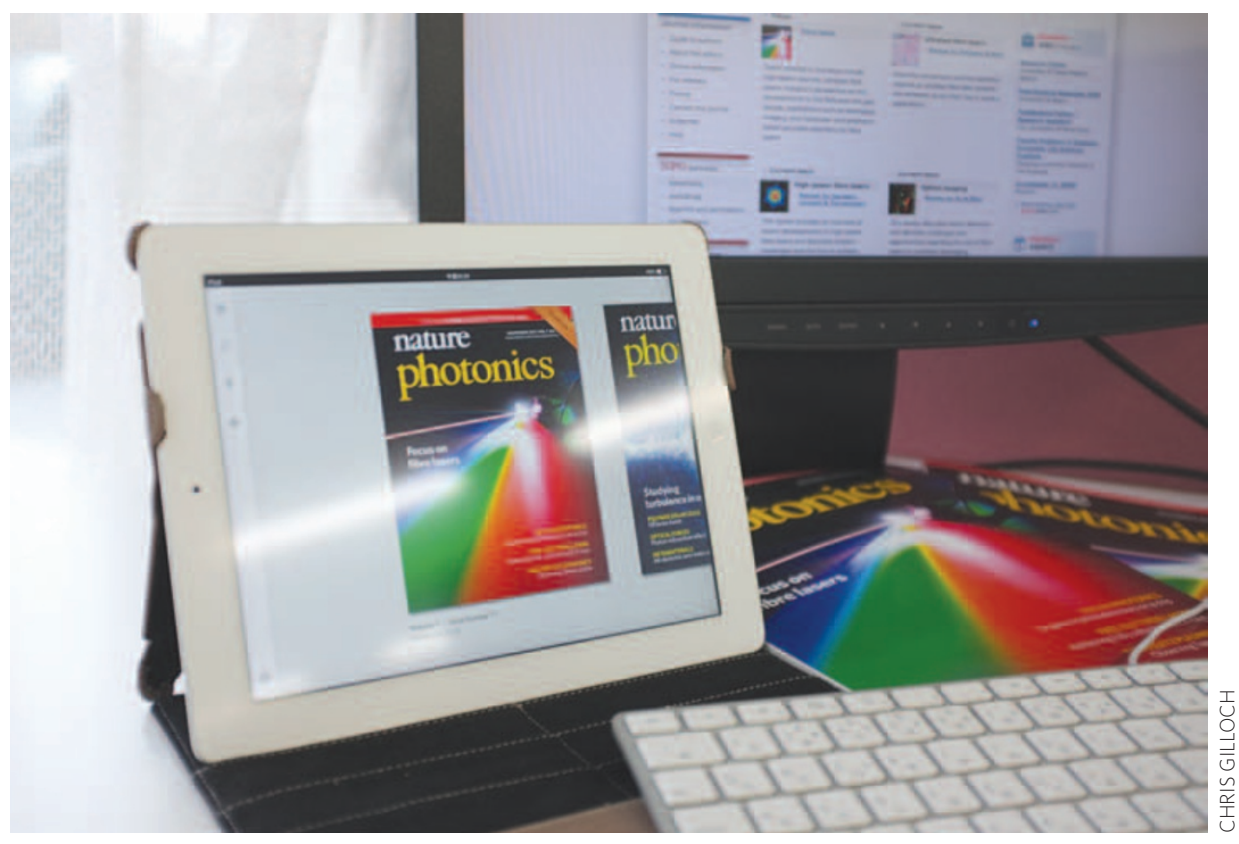

access to the latest papers published in IOPowned journals, including the Journal of Optics, New Journal of Physics, Measurement Science and Technology and Semiconductor Science and Technology. The latest 25 papers published across all journals, in a particular subject category or in a specific journal are listed, and they can be browsed and downloaded. IOPscience Express allows up to 20 articles per month to be downloaded.

The American Institute of Physics (AIP), the publisher of Applied Physics Letters, Journal of Applied Physics and other journals, has adopted a different and innovative approach. As well as providing an app for subscribers called iResearch for browsing and downloading papers in AIP journals (users simply need to log in to their institution's Wi-Fi network to gain access), AIP has also released an app for authors and reviewers. iPeerReview allows prospective authors and reviewers to view papers they are involved with and that are under consideration for publication in an AIP journal. In addition, they can check the status of the papers and download them onto an iOS device for reading offline.

Societies have also been getting in on the act. Although not having a dedicated app for its journals, the Optical Society of America (OSA) does have one called OPN for its member magazine Optics \& Photonics News. After signing in with their subscriber details, readers can download the current issue of the magazine and access an archive of back issues or search for articles of interest. Meanwhile, SPIE, a large society for professionals in photonics, offers apps for quite different reasons. It offers four apps, one (SPIE Conferences) for its conference program, another (SPIE Newsroom) for subscribing to a newsfeed and a third (SPIE Profiles) for accessing its profile database of its members, allowing experts to be found. The fourth (Field Guide to Geometrical Optics) serves as a useful portable reference work complete with data and equations for many common tasks in optical system design, including calculations involving paraxial or Gaussian imaging, aberrations and chromatic effects.

If the latest European Conference on Optical Communication in London this autumn is a reliable indicator of future trends, the days of having to heave bulky, heavy proceedings around at a conference may now be over. The conference app made such a paper copy largely superfluous. This proliferation of apps for those working in photonics brings not only greater convenience but also a more effective means of staying up to date with the latest developments in the world of optics. 